\title{
Research on the correlation of serum plasminogen activator inhibitor-1 level to vascular complications in type 2 diabetes mellitus patients with overweight or obesity
}

\author{
Jun X,'Yanan Z, Zhijie C, Zhihui D, Danhua S, Xiaojing C, Ying W, Jixiang J \\ Department of Geriatrics, the Third Affiliated Hospital of Inner Mongolia Medical University, Baotou, Inner Mongolia, China
}

Received: May 3, 2019

DOI: $10.5430 /$ dcc.v6n2p20
Accepted: June 5, 2019

Online Published: June 10, 2019

\begin{abstract}
Objective: To explore the relationship between serum plasminogen activator inhibitor (PAI-1) level and Type 2 Diabetes Mellitus (T2DM) accompanied by overweight or obesity by observing not only the changes of PAI-1 level in T2DM patients with overweight or obesity, but also glucose and lipid metabolism related indicators, the changes of the inflammatory cytokines secreted by adipocytes, and then making an analysis on the correlation to PAI-1.

Methods: 36 cases of healthy examinees were selected as normal control group (NC group), and the experimental group can be divided into T2DM group (54 cases), Overweight/Obesity group (35 cases) and T2DM + Overweight/Obesity group (48 cases). Glucose and lipid metabolism related indicators such as fasting blood glucose (FBG), triglyceride (TG), total cholesterol (TC), low density lipoprotein cholesterol (LDL-C), glycated hemoglobin (HbA1c), fasting insulin (FINS), insulin resistance index (IR), body weight index (BMI) and inflammatory cytokines (interleukin-6 (IL-6), tumor necrosis factor (TNF- $\alpha$ ) and PAI-1 were observed and compared between groups, and then made an analysis to explore the correlation of these factors to PAI-1.

Results: (1) Compared with NC group, the levels of FBG, HbA1c, FINS and IR were increased in T2DM group, and the difference was of statistical significance. However, there was no statistically significant difference in TG, TC, LDL-C and BMI between NC group and T2DM group; the levels of FINS, IR, TG, LDL-C, TC and BMI were elevated in Overweight/Obesity group, and the difference was of statistical significance. However, there was no statistically significant difference in FBG and HbA1c; the levels of FBG, HbA1c, FINS, IR, TG, LDL-C, TC and BMI were up-regulated in T2DM + Overweight/Obesity group, and the difference was of statistical significance. Compared with T2DM group, the levels of TG, TC, LDL-C and BMI were increased in Overweight/Obesity group, and the difference was of statistical significance, however, the levels of FBG, HbA1c, FINS and IR were decreased, and the difference was statistically significant; The levels of FINS, IR, TG, TC, LDL-C and BMI were elevated in T2DM + Overweight/Obesity group, and the difference was of statistical significance, however, there was no statistically significant difference in FBG and HbA1c. Compared with Overweight/Obesity group, the levels of FBG, FINS, IR, HbA1c and LDL-C were increased in T2DM + Overweight/Obesity group, and the difference was of statistical significance. However, the difference in TG, TC and BMI was not statistically significant. (2) Compared with NC group, the levels of IL-6, TNF- $\alpha$ and PAI-1 were increased in T2DM group, Overweight/Obesity group and T2DM + Overweight/Obesity group, and the difference was statistically significant. Compared with T2DM group, the levels of IL-6 and TNF- $\alpha$ were elevated in Overweight/Obesity group, and the difference was of statistical significance, but there was no statistically significant difference in PAI-1; the levels of IL-6, TNF- $\alpha$ and PAI-1 were up-regulated in T2DM + Overweight/Obesity group, and the difference
\end{abstract}

*Correspondence: Jun X; Email: nmgbgyyyy@163.com; Address: Department of Geriatrics, the Third Affiliated Hospital of Inner Mongolia Medical University, Baotou, Inner Mongolia 014010, China. 
was statistically significant. Compared with Overweight/Obesity group, there was no statistically significant difference in IL-6 and TNF- $\alpha$ between T2DM + Overweight/Obesity group and Overweight/Obesity group, but the level of PAI-1 was increased in T2DM + Overweight/Obesity group, and the difference was of statistical significance. (3) Multivariate Logistic Regression Analysis showed that HbA1c, IR, TG, BMI, IL-6 and TNF- $\alpha$ were independently associated with the level of PAI-1 (all $p<.05$ ). Conclusions: (1) The level of PAI-1 is higher in type 2 diabetes mellitus patients with overweight or obesity than that in patients only with type 2 diabetes mellitus, and it is one of causes that result in vascular complications. (2) The increase in the level of PAI- 1 is considered to be associated with IL- 6 and TNF- $\alpha$ secreted by adipocytes.

Key Words: Plasminogen activator inhibitor-1, Type 2 diabetes mellitus, Overweight/obesity

\section{INTRODUCTION}

According to the statistical results published by International Diabetes Federation (IDF) in 2015, about 415 million people suffer from diabetes mellitus, and another 318 million people develop impaired glucose tolerance. The latter population will also suffer from diabetes eventually. Therefore, it is estimated that the number of people who suffer from diabetes will rise up to 642 million in 2040. China ranked first in the top ten countries with the largest number of diabetics all over the world in 2015, with the number of diabetic patients reaching up to 109.6 million. ${ }^{[1,2]}$ Following various tumors and cardiovascular diseases, diabetes mellitus has been a non-infectious chronic disease which threatens human health, increases social and people's financial burdens and lowers people's life quality. Vascular complication is the most commonly-seen and the most serious disease among various diabetic complications. It not only has a greatest effect, but also is a main factor that leads to the death and disability in diabetics. Overweight or obesity is closely associated with type 2 diabetes mellitus, weight gain is an independent risk factor for the occurrence and development of T2DM. The incidence of diabetes in Chinese overweight patients is $12.8 \%$, and the incidence in the obese patients is $18.5 \%{ }^{[2,3]}$ Meanwhile, some researches show that diabetics with overweight or obesity are more susceptible to vascular complications than patients only with diabetes mellitus. ${ }^{[4]}$ Therefore, it is crucial to focus and research the factors for vascular complications in T2DM patients, especially in those with overweight or obesity, in order to prevent the occurrence of cardiovascular events and reduce mortality.

One of common mechanisms that lead to the occurrence of diabetic vascular complications is the imbalance of the fibrinolytic system. Physiologically, fibrins formed in the body are eliminated by fibrinolysins, which are prevented from over-activation by the antifibrinolytic system, so that the fibrinolytic process and the antifibrinolytic process in the body can be maintained in a dynamic balance. The increase in PAI-1 can lead to the depression of local fibrinolytic activity and the decrease in the degradation of fibrins, inducing the occurrence of thrombosis. The increase in PAI-1 level is a main indicator used to predict the occurrence of cardiovascular events. This research is designed to explore the relationship between PAI-1 level and Type 2 Diabetes Mellitus (T2DM) accompanied by overweight or obesity by observing not only the changes of PAI-1 level in T2DM patients with overweight or obesity, but also the changes of glucose and lipid metabolism related indicators as well as the inflammatory cytokines secreted by adipocytes, and then provide a theoretical basis for the clinical prevention in the occurrence and development of diabetic cardiovascular complications.

\section{RESEARCH OBJECTS AND METHODS}

\subsection{Research objects}

\subsubsection{Normal control group (NC group)}

36 cases of healthy examinees (aged from 35 to 60, mean age of $45.80 \pm 8.0, \mathrm{BMI}<24 \mathrm{~kg} / \mathrm{m}^{2}$ ) were randomly selected in Physical Examination Department of the Third Affiliated Hospital of Inner Mongolia Medical University from December of 2016 to June of 2017, with no history of diabetes, cardiovascular and cerebrovascular diseases as well as liver and renal diseases.

\subsubsection{T2DM group}

54 cases of T2DM patients (aged from 35 to 60, mean age of $46.50 \pm 7.8$, BMI $<24 \mathrm{~kg} / \mathrm{m}^{2}$ ) admitted to Outpatient Department of the Third Affiliated Hospital of Inner Mongolia Medical University during the same period were selected, and the diagnosis of T2DM was consistent with the diagnostic standards established by WHO in 1999; these patients had no diabetic acute or chronic complications, with HbA1c no more than $8.5 \%$, and had no history of cardiovascular and cerebrovascular diseases as well as liver and renal diseases.

\subsubsection{Overweight/Obesity group}

35 cases of overweight/obese patients (aged from 35 to 60 , mean age of $45.80 \pm 6.5, \mathrm{BMI} \geq 24 \mathrm{~kg} / \mathrm{m}^{2}$ ) admitted to Outpatient Department of the Third Affiliated Hospital of Inner Mongolia Medical University during the same period were selected. The diagnosis of obesity was consistent with "Chinese guidelines for the prevention and control of overweight 
and obesity in adults (Trial)" (2003).

\subsubsection{T2DM + Overweight/Obesity group}

48 cases of T2DM patients with overweight/obesity (aged from 35 to 60 , mean age of $46.80 \pm 7.5, \mathrm{BMI} \geq 24 \mathrm{~kg} / \mathrm{m}^{2}$ ) admitted to Outpatient Department of the Third Affiliated Hospital of Inner Mongolia Medical University during the same period were selected. The diagnosis of obesity was consistent with "Chinese guidelines for the prevention and control of overweight and obesity in adults (Trial)" (2003); these patients had no diabetic acute or chronic complications, with $\mathrm{HbA} 1 \mathrm{c}$ no more than $8.5 \%$, and had no history of cardiovascular and cerebrovascular diseases as well as liver and renal diseases.

\subsection{Methods}

\subsubsection{Glucose and lipid metabolism related indicators}

The biochemical method was used to detect the levels of FBG, TG, TC, LDL-C, HbA1c and FINS; height and weight were measured to calculate BMI: BMI = weight $/$ height ${ }^{2}\left(\mathrm{~kg} / \mathrm{m}^{2}\right)$; homeostasis model assessment - insulin resistance index was calculated: $($ HOMA-IR $)=$ FBG $\times$ FINS/22.5.

\subsubsection{Inflammatory cytokines}

Radio immunoassay was used to measure the levels of IL-6 and TNF- $\alpha$.

\subsubsection{ELISA (Enzyme Linked Immunosorbent Assay Double-Antibody Sandwich Technique)}

ELISA was used to measure the level of PAI-1.

\subsection{Statistical analysis}

All above-mentioned indicators were the measurement data, which were represented by $\bar{X} \pm$ s. SPSS 22.0 software was applied to statistical analysis, which showed that the experimental data in each group were fit to normal distribution and homogeneity. One-way ANOVA and LSD test were used in the comparison between two groups, and the difference $p \leq$ .05 was of statistical significance. The correlation analysis was made between PAI-1 and other variates to establish a multivariate linear regression model aiming at related variates, and the difference $p \leq .05$ was statistically significant.

\section{Results}

3.1 The comparison in glucose and lipid metabolism related indicators between each group of patients Compared with NC group, the levels of FBG, HbA1c, FINS and IR were increased in T2DM group, and the difference was of statistical significance $(p<.05)$, but there was no statistically significant difference in TG, TC, LDL-C and BMI between NC group and T2DM group $(p>.05)$; the levels of FINS, IR, TG, LDL-C, TC and BMI were elevated in Overweight/Obesity group, and the difference was of statistical significance $(p<.05)$, however, there was no statistically significant difference in FBG and HbA1c $(p>.05)$; the levels of FBG, HbA1c, FINS, IR, TG, LDL-C, TC and BMI were up-regulated in T2DM + Overweight/Obesity group, and the difference was of statistical significance $(p<.05)$. Compared with T2DM group, the levels of TG, TC, LDL-C and BMI were increased in Overweight/Obesity group, and the difference was of statistical significance $(p<.05)$. However, the levels of FBG, HbAlc, FINS and IR were decreased, and the difference was statistically significant $(p<.05)$. The levels of FINS, IR, TG, TC, LDL-C and BMI were elevated in T2DM + Overweight/Obesity group, and the difference was of statistical significance $(p<.05)$, however, there was no statistically significant difference in FBG and HbA1c $(p>$ .05). Compared with Overweight/Obesity group, the levels of FBG, FINS, IR, HbA1c and LDL-C were increased in $\mathrm{T} 2 \mathrm{DM}+$ Overweight/Obesity group, and the difference was of statistical significance $(p<.05)$. However, the difference in TG, TC and BMI was not statistically significant $(p>.05)$ (see Table 1).

\subsection{The comparison in inflammatory cytokines and PAI- 1 between each group of patients}

Compared with NC group, the levels of IL-6, TNF- $\alpha$ and PAI-1 were increased in T2DM group, Overweight/Obesity group and T2DM + Overweight/Obesity group, and the difference was of statistically significant $(p<.05)$. Compared with T2DM group, the levels of IL- 6 and TNF- $\alpha$ were elevated in Overweight/Obesity group, and the difference was of statistical significance $(p<.05)$, but there was no statistically significant difference in PAI-1 $(p>.05)$; the levels of IL-6, TNF- $\alpha$ and PAI-1 were up-regulated in T2DM + Overweight/Obesity group, and the difference was statistically significant $(p<.05)$. Compared with Overweight/Obesity group, there was no statistically significant difference in IL-6 and TNF- $\alpha$ between T2DM + Overweight/Obesity group and Overweight/Obesity group $(p>.05)$, but the level of PAI-1 was increased in T2DM + Overweight/Obesity group, and the difference was of statistical significance $(p<.05)$ (see Table 2).

\subsection{Correlation analysis of PAI-1 and other factors}

Taking PAI-1 as a dependent variable and FBG, FINS, IR, $\mathrm{HbA} 1 \mathrm{c}$ and other suspicious factors as independent variables, univariate analysis ( $t$-test or nonparametric test) was used to screen out 8 suspicious influencing factors with statistical significance. Later, Logistic regression analysis was performed to conditionally filter variables step by step. According to entry criteria of $0.05, \mathrm{HbA} 1 \mathrm{c}$, IR, TG, BMI, IL- 6 and TNF- $\alpha$ were entered into the equation (see Tables 3-4). 
Table 1. The comparison in glycometabolism related indicators between each group of patients $(\bar{X} \pm \mathrm{s})$

\begin{tabular}{|c|c|c|c|c|c|c|c|c|}
\hline Group & $\begin{array}{l}\text { FBG } \\
(\mathrm{mmol} / \mathrm{L})\end{array}$ & $\begin{array}{l}\text { TG } \\
(\mathrm{mmol} / \mathrm{L})\end{array}$ & $\begin{array}{l}\text { TC } \\
(\mathrm{mmol} / \mathrm{L})\end{array}$ & $\begin{array}{l}\text { LDL } \\
(\mathrm{mmol} / \mathrm{L})\end{array}$ & $\begin{array}{l}\text { FINS } \\
(\mathrm{u} \mathrm{IU} / \mathrm{ml})\end{array}$ & IR & $\begin{array}{l}\text { HbA1c } \\
(\%)\end{array}$ & $\begin{array}{l}\text { BMI } \\
\left(\mathrm{kg} / \mathrm{m}^{2}\right)\end{array}$ \\
\hline NC Group & $5.18 \pm 0.66$ & $1.54 \pm 0.56$ & $4.19 \pm 0.86$ & $2.37 \pm 0.39$ & $12.73 \pm 3.79$ & $3.01 \pm 1.24$ & $4.85 \pm 0.5$ & $21.89 \pm 1.29$ \\
\hline T2DM Group & $8.71 \pm 1.0^{\Delta}$ & $1.63 \pm 0.66$ & $4.36 \pm 1.01$ & $2.53 \pm 0.42$ & $21.9 \pm 3.40^{\Delta}$ & $8.52 \pm 1.32^{\Delta}$ & $8.01 \pm 0.6^{\Delta}$ & $21.58 \pm 1.23$ \\
\hline $\begin{array}{l}\text { Overweight/ } \\
\text { Obesity Group }\end{array}$ & $5.01 \pm 0.7^{*}$ & $3.2 \pm 0.7 \Delta^{*}$ & $5.4 \pm 0.1^{\Delta^{*}}$ & $3.6 \pm 0.2^{\Delta^{*}}$ & $16.2 \pm 3.8^{\Delta^{*}}$ & $4.8 \pm 1.6^{\Delta^{*}}$ & $4.05 \pm 0.4^{*}$ & $26.76 \pm 1.3^{\Delta^{*}}$ \\
\hline $\begin{array}{l}\text { T2DM+ } \\
\text { Overweight/ } \\
\text { Obesity Group }\end{array}$ & $8.73 \pm 1.1^{\Delta^{\text {th }}}$ & $3.3 \pm 0.7 \Delta^{*}$ & $5.5 \pm 0.9 \Delta^{*}$ & 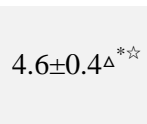 & $7.87 \pm 4.44^{* \text { 访 }}$ & $11.51 \pm 1.7 \Delta^{* \text { 约 }}$ & $8.32 \pm 0.4^{\Delta}$ & $27.87 \pm 1.1^{\Delta^{*}}$ \\
\hline
\end{tabular}

Table 2. The comparison in inflammatory cytokines and PAI-1 between each group of patients $(\bar{X} \pm \mathrm{s})$

\begin{tabular}{llll}
\hline Group & IL-6 $(\mathbf{p g} / \mathbf{m l})$ & TNF- $\boldsymbol{\alpha}(\mathbf{n g} / \mathbf{m l})$ & PAI-1 (ng/ml) \\
\hline NC Group & $75.35 \pm 47.75$ & $0.72 \pm 0.39$ & $23.48 \pm 2.94$ \\
T2DM Group & $110.58 \pm 39.53^{\Delta}$ & $1.12 \pm 0.43^{\Delta}$ & $33.59 \pm 3.86^{\Delta}$ \\
Overweight/Obesity Group & $140.56 \pm 39.53^{*}$ & $1.79 \pm 0.44 \Delta^{*}$ & $32.40 \pm 3.70^{\Delta}$ \\
T2DM + & $148.89 \pm 44.34^{*}$ & $1.83 \pm 0.34 \Delta^{*}$ & $47.10 \pm 4.14 \Delta^{*}$ \\
\hline
\end{tabular}

Note. Compared with NC group, ${ }^{\Delta} p<.05$; Compared with T2DM group, ${ }^{*} p<.05$; Compared with Overweight/Obesity group, ${ }^{\overrightarrow{3}} p<.05$.

Table 3. Correlation analysis of PAI-1 and other factors

\begin{tabular}{lllllllllll}
\hline & FBG & FINS & IR & HbA1c & TG & TC & LDL & BMI & IL-6 & TNF- $\alpha$ \\
\hline Correlation Coefficient $r$ & 0.01 & 0.29 & 0.64 & 0.42 & 0.54 & 0.10 & 0.36 & 0.77 & 0.71 & 0.64 \\
$p$ & .941 & .003 & $<.001$ & $<.001$ & $<.001$ & $<.331$ & $<.001$ & $<.001$ & $<.001$ & $<.001$ \\
\hline
\end{tabular}

Table 4. Multivariate linear regression analysis of PAI-1 and other correlative factors

\begin{tabular}{llllllllll}
\hline & Constant & FINS & IR & HbA1c & TG & LDL & BMI & IL-6 & TNF- $\alpha$ \\
\hline B Value & -15.67 & 0.04 & 0.43 & 2.07 & 0.93 & 0.18 & 0.82 & 0.06 & 1.63 \\
T Value & -3.35 & 0.62 & 2.49 & 3.74 & 2.60 & 0.28 & 5.26 & 7.83 & 2.40 \\
$p$ & .001 & .538 & .014 & $<.001$ & .011 & .779 & $<.001$ & $<.001$ & .018 \\
\hline
\end{tabular}

\section{Discussion}

T2DM is a chronic progressive disease, accompanied by the increased morality of endothelial dysfunction and cardiovascular events. The subsequent vascular complications are main factors that lead to the death and disability in diabetics. Meanwhile, it is shown that T2DM patients with overweight/obesity have a higher incidence of vascular complications. PAI-1 primarily comes from viscera, subcutaneus adipose tissue, vascular endothelia and hepar. The imbalance of the fibrinolytic system resulting from the increased level of PAI- 1 is one of common mechanisms that lead to the occurrence of diabetic vascular complications.

The results in this research show that the level of PAI-1 is higher both in T2DM group and Overweight/Obesity group than that in NC group, and it is much higher in T2DM + Overweight/Obesity group than that in T2DM group. Therefore, PAI-1 is considered to be one of causes that make T2DM pa- tients or T2DM patients with overweight/obesity susceptible to vascular complications. Multivariate Logistic Regression Analysis shows that IR, HbA1c, TG, BMI, IL- 6 and TNF- $\alpha$ are independently correlated to the level of PAI-1. Hence, the increase in the level of PAI-1 is considered to be associated with persistent hyperglycemia, glucose and lipid metabolism disorder and insulin resistance in T2DM patients. In addition, the level of PAI-1 is higher in T2DM + Overweight/Obesity group than that in T2DM patients, which is considered to be related with the increase in the inflammatory cytokines (IL-6 and TNF- $\alpha$ ) secreted by adipocytes.

\subsection{The analysis on the cause of the increase in PAI-1 in T2DM patients}

This research shows that the level of PAI-1 is higher in T2DM group than that in NC group, and Multivariate Logistic Regression Analysis shows that the level of PAI-1 is correlated to IR, HbA1c and TG. Therefore, it is considered that persis- 
tent hyperglycemia, glucose and lipid metabolism disorder and insulin resistance in T2DM patients can lead to the increased level of PAI-1.

PAI-1 primarily comes from vascular endothelia, whose injury can result in the increased secretion of PAI-1. On the one hand, hyperglycemia can directly lead to vascular endothelial injury and then result in the increased secretion of PAI-1. On the other hand, it can also damage vascular endothelia by promoting the formation of advanced glycation end products (AGEs) inside and outside the cells. AGEs can promote the generation of reactive oxygen species and induce peroxidation reaction by modifying proteins, lipids and nucleic acids to damage vascular endothelia.

The up-regulate of HbA1c decelerates the oxygen dissociation of hemoglobins, decreases the oxygenation activity and significantly reduces the level of 2, 3-diphosphoglycerate, leading to histogenous hypoxia and causing vascular endothelial injury.

The rise of TG can lead to the increase in free fatty acids (FFA), which can directly stimulate endothelial cells to generate PAI-1. In addition, serum TG can lead to the significant increase in PAI-1 level by improving the secretion of inflammatory mediators in patients with non-alcoholic fatty liver disease (NAFLD). ${ }^{[5,6]}$

On the condition of insulin resistance, a high level of insulin can directly damage vascular endothelial function by increasing endothelin-1 (ET-1) and reducing the level of folic acids. ${ }^{[7]}$ ET-1 is a type of endogenous vasoconstriction polypeptide which vascular endothelial cells secrete the most. It has a most lasting effect, and its up-regulate can disorder the endothelial function. On the one hand, folic acids can minimize vascular endothelial injury caused by homocysteinemia by decreasing the level of homocysteine (Hcy); on the other hand, it can regulate the degree of gene methylation which is related with peroxidation reaction to affect gene expression and reduce vascular endothelial injury resulting from peroxidation reaction. ${ }^{[8]}$ Therefore, hyperinsulinemia is often accompanied by the increased level of PAI-1. ${ }^{[9]}$

To sum up, hyperglycemia, glucose and lipid metabolism disorder and insulin resistance occurring in T2DM patients can promote endothelial cells to secrete more PAI-1 by damaging vascular endothelial cells.

\subsection{The analysis on the cause of the increase in PAI-1 by obesity}

The results in this research show that the level of PAI-1 is increased both in T2DM + Overweight/Obesity group and NC group. Meanwhile, Multivariate Logistic Regression Analysis shows that the level of PAI-1 is correlated to IL-6 and TNF- $\alpha$. Therefore, it is considered that the obvious increase in PAI-1 level in T2DM patients with overweight/obesity is associated with inflammatory cytokines (IL-6 and TNF- $\alpha$ ) secreted by adipocytes. In recent years, adipose tissue has been re-recognized as an endocrine organ. Especially its secreted cytokines, play an important role in the occurrence and development of cardiovascular events. Overweight or obese patients stay in a low-degree chronic inflammatory state for a long term, the levels of IL-6, TNF- $\alpha$, CRP and other inflammatory cytokines in those patients are obviously increased. ${ }^{[10]}$ Some researches find that, ${ }^{[11]}$ if TNF- $\alpha$ is used to simulate peritoneal inflammation state and interfere with the balance of fibrinolytic system and antifibrinolytic system, the level of PAI-1 generated by peritoneal mesothelial cells will be significantly increased. With the addition of simvastatin to depress TNF- $\alpha$, the expression of PAI- 1 is significantly decreased. IL-6 makes leukocytes adhere to the surface of endothelial cells by stimulating the expression of intercellular adhesion molecules. Furthermore, by inducing the expression of chemokines, it recruits a large number of inflammatory cells to make endothelial cells develop inflammatory reactions, damage endothelial cells and improve the secretion of PAI-1. ${ }^{[12,13]}$

\section{Conclusion}

It is considered that the increase in the levels of inflammatory cytokines (IL-6 and TNF- $\alpha$ ) in patients with overweight/obesity leads to the increase in the expression of PAI-1. Compared with T2DM patients, T2DM patients with overweight/obesity have a greater risk for diabetic vascular complications. It is important to reduce and control body weight when effectively regulating the blood glucose level.

\section{CONFLICTS OF INTEREST DISCLOSURE}

The authors declare they have no conflicts of interest.

\section{REFERENCES}

[1] Ogurtsova K, Da rocha fernandes JD, Huang Y, et al. Idf diabetes atlas: global estimates for the prevalence of diabetes for 2015 and 2040. Diabetes Res Clin Pract. 2017; 128: 40-50. PMid:28437734. https://doi.org/10.1016/j.diabres.2017.03.024

[2] Yang W, Lu J, Weng J, et al. Prevalence of diabetes among men and women in china. N Engl J Med. 2010; 362(12): 1090-1101. PMid:20335585. https://doi.org/10.1056/NEJMoa0908292 
[3] Schwartz S, Fabricatore AN, Diamond A. Weight reduction in diabetes. Adv Exp Med Biol. 2012; 771: 438-458. PMid:23393695. https : //doi.org/10.1007/978-1-4614-5441-0_31

[4] Jensen MD, Ryan DH, Apovian CM, et al. 2013aha/acc/tos guideline for the management of overweight and obesity in adults: a report of the American college of cardiology/American heart association task force on practice guidelines and the obesity society. J Am Coll Cardiol. 2014; 63(25): 2985-3023. https ://doi.org/10.1016/ j.jacc. 2013.11.004

[5] Xiuping B, Baozhen Z, Aiqing L, et al. The influence of serum levels of triglicerides and uric acids on insulin resistance and tumor necrosis factor alpha and interleukin-6 in patients with nonalcoholic fatty liver disease. Chinese Journal of Gastroenterology and Hepatology. 2012; 21(10): 936-938.

[6] Xiuping B, Yanlan Y, Min G, et al. The influence of serum triglycerides on plasminogen activator inhibitor-1 and adiponectin in patients with nonalcoholic fatty liver disease. Chinese Journal of Gastroenterology and Hepatology. 2014; 23(4): 435-437.

[7] Shinozaki K, Hirayama A, Nishio Y, et al. Coronary endothelial dysfunction in the insulin-resistant state is linked to abnormal pteridine metabolism and vascular oxidative stress. J Am Coll Cardiol. 2001; 38(7): 1821-1828. https ://doi.org/10.1016/S0735-1097(01 ) 01659-X

[8] Da silva RP, Kelly KB, AL rajabi A, et al. Novel insights on interactions between folate and lipid metabolism. Biofactors. 2014; 40(3):
277-283. PMid:24353111. https ://doi .org/10.1002/biof . 1 154

[9] Furumoto T, Fujii S, Nishihara K, et al. Maladaptive arterial remodeling with systemic hypertension associated with increased concentrations in blood of plasminogen activator inhibitor type-1 (pai-1). Am J Cardiol. 2004; 93(8): 997-1001. PMid:15081442. https ://doi.org/10.1016/j.amjcard.2003.12.053

[10] Cesari M, Pahor M, Incalzi RA. Plasminogen activator inhibitor-1 (pai-1): a key factor linking fibrinolysis and age-related subclinical and clinical conditions. Cardiovasc Ther. 2010; 28(5): 72-91. PMid:20626406. https : //doi .org/10.1111/j.1755-5922.20 10.00171.x

[11] Gong T, Jinsong H. Effects of simvastatin on TNF- $\alpha$-induced t-PA and PAI-1 expression in human peritoneal mesothelial cells in vitro. Journal of Clinical Nephrology. 2011; 5(4): 239-243.

[12] Souza JR, Oliveira RT, Blotta MH, et al. Serum levels of interleukin6 (Il-6), Interleukin-18 (Il-18) and C-reactive protein (crp) in patients with type-2diabetes and acute coronary syndrome without ST-segment elevation. Arq Bras Cardiol. 2008; 90(2): 86-90. PMid:18392379. https://doi.org/10.1590/S0066-782X200 8000200004

[13] Bihui Q, Tao H, Min H, et al. Alteration of interleukin 6 level in development of deep vein thrombosis in rats. Chinese Journal of General Surgery. 2014; 23(6): 765-768. 